\title{
Metabolismo en los edificios. Estudio de medidas para el ahorro del consumo de agua en tres centros docentes en Madrid
}

\section{Buildings metabolism. Study of measures to save water consumption in three schools in Madrid}

\author{
J. Gallego Sánchez-Torija ${ }^{(*)}$, E. Larrumbide Gómez-Rubiera ${ }^{(* *)}$, C. Bedoya Frutos ${ }^{(*)}$
}

\section{RESUMEN}

Estudios previos han investigado sobre el desempeño energético de centros docentes. Sin embargo, no parece haberse explorado suficientemente la relación entre consumo de energía y consumo de agua en este tipo de edificios. Este artículo presenta los resultados de un estudio empírico, basado en la metodología de la auditoría energética operativa, realizado en tres centros docentes ubicados en la Comunidad de Madrid. Los resultados sugieren que las medidas de ahorro en el consumo de agua, como implantar perlizadores, temporizadores o doble pulsadores, son relevantes en cuanto a la mejora de la eficiencia energética, suponen inversiones moderadas y obtienen unos periodos de retorno simple de la inversión atractivos para los gestores de los edificios. Se abren nuevas vías de investigación que apuntan a realizar el análisis de otros flujos de materia e información, de manera que se establezca la relación entre dichos flujos y el consumo de energía que llevan aparejados.

Palabras clave: agua, eficiencia energética, sostenibilidad, auditoría energética, metabolismo arquitectónico, centros docentes, análisis de ciclo de vida, pobreza energética.

\section{ABSTRACT}

Recent research has studied the energy performance of buildings for educational use. However, the relationship between energy consumption and water consumption in this type of buildings does not seem to have been sufficiently explored. This paper presents results of an empirical research, based on the methodology of the operational energy audit, carried out in three buildings for educational use located in Madrid Community. Findings suggest that the water saving measures, such implant pearlizers, timers or double push buttons, are relevant in terms of improving energy efficiency, they suppose moderate investments and they obtain simple return periods of investment attractive to the managers of the buildings. New avenues of research are opened that aim to carry out the analysis of other flows of matter and information, so as to establish the relationship between these flows and the consumption of energy that they suppose.

Keywords: water, energy efficiency, sustainability, energy audit, architectural metabolism, buildings for educational use, life cycle analysis, fuel poverty.

(*) Departamento de Construcción y Tecnología Arquitectónicas, Escuela Técnica Superior de Arquitectura, Universidad Politécnica de Madrid (España).

(**) Instituto Eduardo Torroja de Ciencias de la Construcción. Consejo Superior de Investigaciones Científicas (España). Persona de contacto/Corresponding author: jorge.gallego@upm.es (J. Gallego Sánchez-Torija).

ORCID: https://orcid.org/oooo-0oo2-3240-1883 (J. Gallego Sánchez-Torija); https://orcid.org/oooo-0003-2265-7794 (E. Larrumbide Gómez-Rubiera); https://orcid.org/oooo-0oo1-6756-0713 (C. Bedoya Frutos).

Cómo citar este artículo/Citation: Gallego Sánchez-Torija, J.; Larrumbide Gómez-Rubiera, E.; Bedoya Frutos, C. (2020). Metabolismo en los edificios. Estudio de medidas para el ahorro del consumo de agua en tres centros docentes en Madrid. Informes de la Construcción, 72(557): e325. https://doi.org/10.3989/ic.67829

Copyright: (c) 2020 CSIC. Este es un artículo de acceso abierto distribuido bajo los términos de la licencia de uso y distribución Creative Commons Reconocimiento 4.0 Internacional (CC BY 4.0). 


\section{INTRODUCCIÓN}

La palabra metabolismo se define según el Diccionario de la lengua española como "el conjunto de reacciones químicas que efectúan las células de los seres vivos con el fin de sintetizar o degradar sustancias." (1)

La conceptualización de las ciudades como sistemas naturales y organismos vivos llevó a Abel Colman en 1965 a definir el metabolismo urbano, o metabolismo de las ciudades, como "todos los materiales y materias primas necesarias para mantener a los habitantes de una ciudad, en una casa, en el trabajo y en el juego.” (2)

Dicha definición se ha ido enriqueciendo y ha añadido mayor complejidad a lo largo del tiempo. Kennedy, Cuddihy y Engel-Yan en 2007 definen metabolismo urbano como "la suma total de los procesos técnicos y socioeconómicos que ocurren en las ciudades, resultado del crecimiento, producción de energía y eliminación de desechos.” (3)

En un primer momento se entienden las ciudades como organismos que intercambian materiales, estudiando los flujos de materia que entra en las ciudades y los flujos de desechos que salen de las mismas. Posteriormente el metabolismo urbano estudia los intercambios de materia, energía e información dentro de las ciudades y con el medio circundante (4).

Siguiendo con la analogía organicista, si se entiende la ciudad como organismo, se puede realizar un acercamiento a los edificios como células que forman parte de dicho organismo. De hecho, plantearse los sistemas energéticos a escala de edificio o de barrio, en lugar de a escala de ciudad o de territorio, permitiría aumentar la resiliencia urbana (5), lo que supone una mayor capacidad de adaptación ante escenarios adversos donde el abastecimiento de dichos suministros resulte más difícil.

La arquitectura que se enmarca dentro del sistema productivo dominante sigue el mismo proceso que el resto de objetos de consumo: extracción, fabricación y utilización, para acabar convirtiéndose en un residuo (6). En la biosfera estos procesos siguen una lógica distinta. Los residuos de los procesos humanos, constituyen, en los procesos naturales, recursos que se utilizan para volver a comenzar el ciclo de fabricación y utilización. De esta manera la naturaleza funciona con ciclos circulares a diferencia de los ciclos lineales característicos de la actividad humana convencional (7).

Los flujos de materia y energía en edificación se estudian mediante el análisis del ciclo de vida (ACV). Se trata de una metodología que permite la valoración exhaustiva del impacto ambiental de los edificios, realizando una contabilidad completa del consumo de recursos y de la emisión de residuos asociados a las distintas fases del ciclo de vida (8).

El análisis del ciclo de vida es un criterio adecuado para cuantificar el comportamiento medioambiental de los edificios en todas sus fases: construcción, funcionamiento, mantenimiento y demolición del edificio. Si se quiere estudiar el metabolismo del parque edificado, habría que centrarse en las fases de funcionamiento y mantenimiento, dado que la construcción ya se ha realizado y la demolición dependerá de cómo se construyó en su momento.
En la actualidad, se pretende mejorar la eficiencia energética de los edificios con el objetivo de reducir las emisiones de gases de efecto invernadero. Para ello se busca enfatizar las ventajas de las medidas de mejora en la eficiencia energética, tanto en lo que se refiere a los períodos de retorno de las inversiones, como a la reducción de costes de funcionamiento (9).

También se estudia la relación entre consumo de energía dentro de los edificios y la situación económica de las personas que los utilizan. El concepto de hogar en situación de pobreza energética, cuyo desarrollo analiza Moore (10) a partir de la primera definición conocida de Boardman (11), se entiende como aquellos hogares cuyo gasto en servicios energéticos excede del 10\% de sus ingresos. Se relaciona así el uso de energía en la vivienda, la renta en el hogar y el coste de la energía.

En el concepto de pobreza energética se incluyen los gastos de calefacción, producción de agua caliente sanitaria, iluminación, equipos y cocina (12). Se descarta de esta consideración el gasto en consumo de agua. Incluso aproximaciones más recientes (13), intentan adaptar dicho concepto a los diversos contextos teniendo en cuenta factores regionales de variaciones climáticas, características constructivas y socioeconómicas; pero siguen dejando de lado el uso del agua.

Empieza a haber voces críticas que llaman la atención acerca de las limitaciones del modelo vigente, que analiza únicamente aspectos energéticos: "La evaluación energética de edificios suele cuantificar consumos, ahorros energéticos y económicos, amortizaciones, emisiones de $\mathrm{CO}_{2}$, etc.; sin embargo, existen otros factores, habitualmente no considerados, que pueden ser determinantes en la elección de soluciones para el mejor comportamiento y la eficiencia energética de los edificios y en el bienestar y la calidad de vida de sus usuarios, especialmente en las viviendas (14).”

La limitación de los enfoques basados en la energía, es que descuidan otros factores que influyen en el metabolismo que sí se tienen en cuenta cuando se estudian los intercambios de materia, energía e información que se producen dentro de los edificios. Es posible reducir las emisiones de gases de efecto invernadero cuando se analizan y se mejoran los intercambios de materia y de información en los edificios, no sólo analizando y mejorando los procesos de intercambio de energía. Habría que avanzar en el paradigma de la eficiencia a la ecología, entendiendo los ciclos que se producen dentro de la arquitectura (15).

El presente trabajo avanza en el entendimiento del metabolismo de los edificios. Estudia el uso que se realiza del agua, desde el punto de vista de un intercambio de materia que tiene consecuencias en el uso de la energía y explora las posibilidades de la reducción de gases de efecto invernadero a través del uso de dicho recurso. Para ello se enfatizan las ventajas en cuanto a los períodos de retorno de las inversiones y a la reducción de los costes de funcionamiento.

De hecho, ya hay autores que han abordado la vinculación del agua con la energía (16), la relación entre agua y energía y entre agua y emisiones de gases de efecto invernadero, destacando su importancia a escala urbana (17), y la utilización de energía a lo largo de los grandes sistemas de infraestructura convencionalmente utilizados para la captación, transporte y distribución de agua, además de para el tratamiento de las aguas usadas $(18,19,20)$. 
También hay autores que han estudiado la historia de las plantas de tratamiento de aguas residuales no sólo como infraestructuras urbanas, sino desde el punto de vista de su integración en el ciclo biológico del agua, estudiando la compleja relación entre sociedad y naturaleza. Schneider introduce el concepto de ecosistema industrial como el proceso metabólico de un ecosistema que está explotado para extraer recursos como alimentos, combustibles, materias primas, etc. (21)

Es más, se ha llegado a estudiar cómo el metabolismo de las ciudades es capaz de transformar y configurar la geografía del territorio (22).

Dentro de este marco, se escogen los centros docentes como ámbito concreto donde realizar el estudio, debido fundamentalmente a tres motivos: En primer lugar se trata de edificios que presentan un consumo de agua relevante debido al elevado número de usuarios, pero sin llegar a ser un consumo tan elevado como para diferenciarse del resto de edificios, como podría ser el caso de una piscina; adicionalmente la propiedad está en manos de un único titular, lo que facilita la gestión de la implantación de las medidas que se consideren interesantes; por último, dado el carácter educativo de la actividad que se desarrolla en el edificio, cualquier acción que se emprenda para mejorar el comportamiento energético puede transformar la mentalidad de las personas que lo usan a diario, ayudando a generar conciencia social de los problemas medioambientales a los que se enfrenta la sociedad actual, dotándola de herramientas eficaces para mitigar los efectos adversos del cambio climático (23).

Se considera, por tanto, que la necesidad de este trabajo se justifica desde el impacto que tendría en la sociedad el hecho de aprovechar y aplicar el conocimiento que está investigación genera.

Muchos estudios en colegios se centran en la calidad del aire, en el consumo de energía para mantener unas adecuadas condiciones térmicas, en el confort acústico y lumínico. También hay estudios acerca de la calidad y de la cantidad del agua que se consume en los colegios. Pero no se encuentran artículos que investiguen el uso que se hace del agua en los centros docentes (más allá del agua destinada al consumo humano). Por eso resulta relevante el presente artículo.

De hecho, el consumo medio de agua en los centros docentes es de 4,7 litros al día por usuario, oscilando entre 2 y 11 litros por usuario y día (24). Lo que nos da idea del importante consumo que se produce y de la capacidad de ahorro que hay en los centros docentes con mayor consumo.

\section{DATOS Y METODOLOGÍA}

\subsection{Metodología}

Se utiliza la metodología de una auditoría energética operativa (23). Dicha auditoría contempla tanto el estudio del desempeño energético como el consumo de agua en los edificios. La metodología que se lleva a cabo para estudiar el consumo de agua consta de 5 pasos:

\section{PRIMER PASO}

En primer lugar, se lleva a cabo una recogida de los datos que ofrece la facturación de la compañía suministradora de agua: consumo de agua y coste de dicho consumo. Se obtienen los datos de 5 años para evitar los errores que se pueden producir debido a causas fortuitas como fugas de agua, averías en el contador, pérdida de alguna factura, etc.

\section{SEGUNDO PASO}

En segundo lugar, se lleva a cabo una toma de datos en el edificio. Se establece un inventario de los equipos que consumen agua.

Se averigua el caudal instantáneo de cada grifo. Para ello se dispone de un recipiente de un litro de capacidad y se cronometrará el tiempo de llenado. Posteriormente, se halla el inverso del tiempo cronometrado y el valor resultante será el caudal unitario del grifo en litros por segundo.

También se toma nota del volumen de las cisternas de los inodoros midiendo sus dimensiones para conocer el consumo de agua por cada descarga.

\section{TERCER PASO}

A continuación, se analizan los datos obtenidos en los dos pasos anteriores.

Se hallan los siguientes indicadores que permitan comparar los datos obtenidos con los datos que ofrecen otros edificios de similares características:

- Consumo diario agua por usuario (l/usuario)

- Emisiones anuales $\mathrm{CO}_{2}$ por usuario (kg/usuario)

- Factura anual agua por usuario ( $€ /$ usuario)

Se compara el valor de los indicadores obtenidos con los indicadores de referencia.

El consumo medio de agua en los centros docentes es de 4,7 litros al día por usuario $(24,25)$.

Para la obtención del indicador de emisiones de $\mathrm{CO}_{2}$ asociadas al consumo de agua se procede de la siguiente manera:

Se aplica un coeficiente de paso de $0,331 \mathrm{kgCO}_{2} / \mathrm{kWh}$ para transformar en emisiones de $\mathrm{CO}_{2}$ la energía eléctrica final necesaria para la captación, depuración y transporte del agua hasta los puntos de consumo. Dicho coeficiente de paso ha sido extraído del documento reconocido para la justificación del cumplimiento de la normativa española (26).

No existe un estudio exhaustivo que determine el consumo energético del ciclo del agua en España (27). Algunos autores aseguran que si al consumo energético realizado en el proceso de depuración de las aguas residuales, se le añade la energía consumida en el transporte de dichas aguas, en la distribución de agua sanitaria desde la captación hasta el consumo y en su captación, la cifra media es de $3 \mathrm{kWh} / \mathrm{m}^{3}$ $(28,29)$. Aunque si el agua para el abastecimiento procede de una desaladora, el consumo unitario energético alcanza los $7,74 \mathrm{kWh} / \mathrm{m}^{3}$ (30).

Con dichos valores, se obtiene que las emisiones de $\mathrm{CO}_{2}$ asociadas al consumo de agua son de $0,331 \mathrm{kgCO}_{2} / \mathrm{kWh} \times 3^{2}$ $\mathrm{kWh} / \mathrm{m}^{3}=0,993 \mathrm{kgCO}_{2} / \mathrm{m}^{3}$. 


\section{CUARTO PASO}

Se establece un reparto del consumo total de agua entre los distintos equipos consumidores existentes en el edificio. Para ello, se estiman los siguientes valores: número de usos diario de los inodoros y de los lavabos por cada usuario del edificio, ratio de litros de agua por cada $\mathrm{m}^{2}$ de edificio, para su limpieza y ratio de litros de agua por cada comida.

Una vez realizadas dichas estimaciones, se halla el cómputo global de consumo que se produce en el edificio y se compara con los datos obtenidos a través de la facturación. Se realiza el ajuste correspondiente de las estimaciones anteriores, de manera que ambos valores coincidan.

Finalmente, a cada grupo de equipos de consumo se cuantifica el gasto económico asociado a su consumo, aplicando los precios del agua que figuran en las facturas recogidas en cada centro docente.

\section{QUINTO PASO}

Se procede al análisis de las propuestas de mejora que se identifican en el edificio. Para ello se cuantifica la reducción de consumo que implica cada propuesta, su ahorro económico, su ahorro de emisiones de $\mathrm{CO}_{2}$, la inversión necesaria para llevarla a cabo y el período de retorno simple de la inversión.

\subsection{Estudio de casos}

El estudio se ha llevado a cabo en tres centros docentes ubicados en la Comunidad de Madrid (figura 1).

- Centro de educación infantil (C.E.I.) La Inmaculada:

- Año de construcción: 2002

- Superficie construida: $870 \mathrm{~m}^{2}$

- Número de alumnos: 180

- Localidad: Madrid

- Zona climática: D3

- Centro de educación infantil y primaria (C.E.I.P.) Regina Assumpta:

- Año de construcción: 1960

- Superficie construida: $2.030 \mathrm{~m}^{2}$

- Número de alumnos: 200

- Localidad: Cercedilla

- Zona climática: E1

- Centro de educación primaria y secundaria (C.E.P.S.) Calasanz:

- Año de construcción: 1970

- Superficie construida: $6.300 \mathrm{~m}^{2}$
- Número de alumnos: 1.000 alumnos

- Localidad: Alcalá de Henares

- Zona climática: D3

Se han seleccionado dichos centros con la intención de cubrir un amplio abanico en cuanto a las características reseñadas (año de construcción, superficie construida, número de alumnos, localidad y zona climática).

En cuanto al tipo de enseñanza, con la muestra seleccionada se cubren los centros de educación infantil, los centros de educación infantil y primaria y los centros de educación primaria y secundaria. Con los centros docentes anteriores, se cubre un $98 \%$ de los tipos de centros educativos existentes en la Comunidad de Madrid, tal y como está recogido en el informe de la Consejería de Educación de la Comunidad de Madrid (31). El 2\% restante que queda sin cubrir se trata de centros específicos de educación especial.

En cuanto al año de construcción, se cubren los centros docentes construidos con anterioridad a la entrada en vigor de la Norma básica de la edificación Condiciones térmicas de 1979 y los construidos al amparo de dicha normativa. Desde este punto de vista, se cubre un $92 \%$ de los centros existentes en la Comunidad de Madrid (32).

En cuanto a la superficie construida se han elegido centros entre $870 \mathrm{~m}^{2}$ y $6.300 \mathrm{~m}^{2}$.

Por lo que respecta al número de alumnos se han elegido centros entre 180 y 1.000 alumnos.

Considerando las localidades, se ha elegido la capital con 3.200.00o habitantes censados en 2011, una localidad grande como Alcalá de Henares con 200.00o habitantes y un pueblo de la sierra como Cercedilla con 7.00o habitantes.

Finalmente, por lo que concierne a la zona climática, se cubren los centros docentes construidos en las zonas climáticas D3 y E1. Se cubre un 97\% de las zonas climáticas donde se ubican los centros existentes en la Comunidad de Madrid.

\subsection{Recogida de datos}

Los datos de facturación con los que se ha trabajado corresponden al periodo de años comprendido entre 2009 y 2013.

La toma de datos de cada edificio descrita en el paso segundo se ha realizado en las siguientes fechas: C.E.I. La Inmaculada el 13 de mayo de 2015, C.E.I.P. Regina Assumpta el 30 de enero de 2015 y C.E.P.S. Calasanz el 3 de junio de 2015.

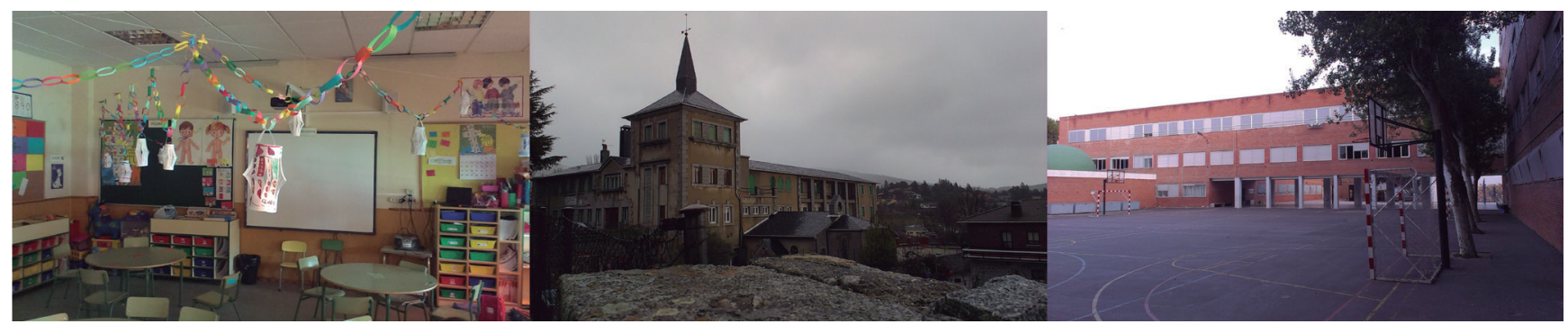

Figura 1. Centro de educación infantil La Inmaculada, Centro de educación infantil y primaria Regina Assumpta y Centro de educación primaria y secundaria Calasanz. 


\subsection{Descripción de las propuestas de mejora y del cálculo del impacto que supone su implantación}

A continuación se procede a describir las propuestas de mejora que se van a estudiar en cada centro docente y el método de cálculo del impacto que supone su implantación.

\section{PROPUESTA DE MEJORA 1: Ecoauditoría del agua.}

El consumo de agua depende directamente de los hábitos de consumo de los usuarios de cada centro docente. Todas las medidas de eficiencia que se estudien, sin un uso adecuado por parte de los usuarios del centro educativo, tendrán un impacto reducido en cuanto al ahorro de agua. Por eso se estudia implantar una ecoauditoría del agua como primera propuesta de mejora para el uso racional del agua.

Con esta medida se involucra a toda la comunidad educativa en la obtención de información acerca de cómo se está efectuando el consumo de agua dentro del edificio. Se potencia un proceso participativo en la toma de decisiones acerca de las medidas a implantar, de su posterior seguimiento de los logros que se van obteniendo y de la búsqueda de recursos para poderlas llevar a cabo, incluso del destino de los recursos que se obtengan con el ahorro de la puesta en marcha de la ecoauditoría.

Para calcular el impacto de esta propuesta, se estable un objetivo no demasiado ambicioso de alcanzar el consumo máximo de los centros educativos de 11 litros por usuario y día (24), en aquellos centros que lo superan. En el resto de centros que están por encima del valor de consumo medio, se considera que con esta medida se puede reducir su consumo en un $20 \%$.

PROPUESTA DE MEJORA 2: Contrapeso en los inodoros con tanque alto.

Los inodoros con tanque alto, cuentan con una cadena para su accionamiento. Cuando esto sucede, se vacía todo el contenido de agua que hay alojado en la cisterna elevada.

Lo que se pretende con la instalación de un contrapeso es detener el vaciado de la cisterna cuando se deja de actuar sobre la cadena. De esta manera, se puede ajustar la cantidad de agua que se utiliza en función de lo que se necesita en cada ocasión, sin verse abocado obligatoriamente a consumir la máxima cantidad de agua en cada ocasión que se usa el inodoro.

Para calcular el impacto de esta propuesta, en primer lugar se mide el volumen de agua contenido en las cisternas de tanque alto existentes. A continuación se estima la diferencia de consumo, considerando que el consumo medio de los inodoros con tanque alto después de la implantación de la medida de 5 litros por descarga. Se trata de una estimación conservadora, ya que cuando es necesaria una descarga completa se utilizan 6 litros y cuando es necesaria una descarga parcial se utilizan 3 litros.

PROPUESTA DE MEJORA 3: Doble pulsador en inodoros con tanque bajo.

Los inodoros con tanque bajo, cuentan con un pulsador para su accionamiento. Cuando se aprieta, se vacía todo el contenido de agua que hay alojado en la cisterna apoyada sobre el inodoro.
Lo que se pretende con la instalación de un doble pulsador es discriminar la cantidad de agua que se va a utilizar según las necesidades. Un pulsador descarga la cisterna completa, mientras que el segundo pulsador realiza una descarga de la mitad de la cisterna. $\mathrm{Al}$ igual que en la propuesta de mejora anterior, se puede ajustar la cantidad de agua que se utiliza en función de lo que se necesita en cada ocasión, sin verse abocado obligatoriamente a consumir la máxima cantidad de agua en cada ocasión que se usa el inodoro.

Para calcular el impacto de esta propuesta, se procede igual que con la propuesta anterior. En primer lugar se mide el volumen de agua contenido en las cisternas de tanque bajo existentes. A continuación se estima la diferencia de consumo, considerando que el consumo medio de los inodoros con tanque alto después de la implantación de la medida de 5 litros por descarga. Se trata, igualmente, de una estimación conservadora, ya que cuando es necesaria una descarga completa se utilizan 6 litros y cuando es necesaria una descarga parcial se utilizan 3 litros.

\section{PROPUESTA DE MEJORA 4: Grifos temporizados.}

Los grifos no temporizados son susceptibles de quedarse abiertos. Esto supondría un despilfarro de agua importante. Sin tener en cuenta este efecto debido al mal uso, se cuantifica el ahorro que supone instalar un grifo temporizado con la tecnología actual que incluye un perlizador en la salida del agua, lo que hace que el caudal que aporta el grifo se disminuya sin perder confort. Además, se propone la instalación de un grifo que se puede regular para que se cierre a los 6 segundos de su accionamiento, lo que reduce el agua que se consume en cada uso frente a los grifos que permanecen abiertos durante 20 segundos.

Para calcular el impacto de esta propuesta, se ha considerando que los grifos no temporizados están abiertos 20 segundos durante cada uso. Si los grifos temporizados se cierran a los 6 segundos, esto supondría una reducción del $70 \%$ del consumo.

Adicionalmente los nuevos grifos incorporan perlizadores que disminuyen su caudal a 2,5 litros por minuto. Se mide el caudal de los grifos existentes para determinar la reducción adicional del consumo debido al perlizador.

A continuación se calcula el efecto combinado de ambas medidas, reducción de tiempo y de caudal.

\section{PROPUESTA DE MEJORA 5: Perlizador en fregaderos.}

Los grifos de los fregaderos actualmente instalados proporcionan un caudal de agua por encima de los 10 litros por minuto.

Se propone incorporar un perlizador que proporcione 4,6 litros por minuto. Se trata de un perlizador específico para fregaderos que cuenta con cabezal y flexo orientable de manera que se pueda enfocar el chorro en todas las direcciones, aportando comodidad de uso a la hora de limpiar los útiles de cocina.

Para calcular el impacto de esta propuesta, se mide el caudal de los grifos existentes en los fregaderos. El caudal con los perlizadores estudiados es 4,6 litros por minuto, con lo que se puede hallar la reducción de consumo que se logra. 


\section{RESULTADOS Y DISCUSIÓN}

\subsection{Resultados}

Los datos de consumo de agua en $\mathrm{m}^{3}$ recogidos de la facturación de los distintos centros se recogen en la tabla 1.

Los datos del coste económico asociados al consumo de agua en $€$ recogidos de la facturación de los distintos centros se recogen en la figura 2.

Los indicadores que se obtienen de los datos anteriores y que permiten comparar el desempeño de los distintos centros, en cuanto al consumo de agua se refiere se muestran en la tabla 2.
La dotación de equipos o sistemas consumidores de agua de cada centro se muestran en la tabla 3.

El reparto del consumo de agua entre los distintos equipos consumidores existentes en cada centro docente, en $\mathrm{m}^{3}$ de consumo anual de agua y su coste asociado en $€$, se realiza según la metodología de la auditoría energética operativa (23), que se explica a continuación:

- Se averigua el consumo medio de los inodoros por cada uso.

- Se averigua el caudal medio de los grifos.

- Se estima el número de usos diario de los inodoros por cada usuario del edificio, teniendo en cuenta el número de horas que permanecen en el mismo.

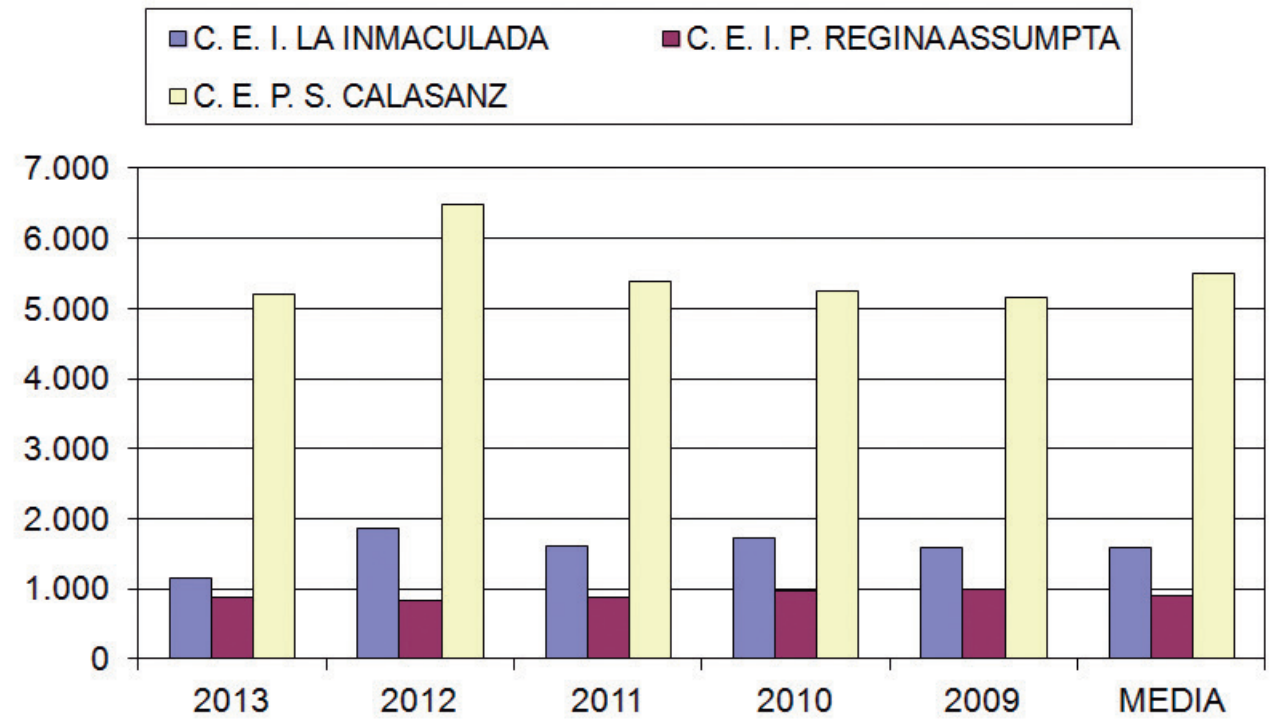

Figura 2. Coste económico asociado al consumo de agua en $€$ de los distintos centros.

Tabla 1. Consumo de agua en $\mathrm{m}^{3}$ de los distintos centros.

\begin{tabular}{|c|c|c|c|c|c|c|}
\hline \multirow[b]{2}{*}{ MESES } & \multicolumn{6}{|c|}{ CONSUMO AGUA $\left(\mathrm{m}^{3}\right)$} \\
\hline & 2013 & 2012 & 2011 & 2010 & 2009 & MEDIA \\
\hline & \multicolumn{6}{|c|}{ C. E. I. LA INMACULADA } \\
\hline ENE-FEB & 129 & 164 & 138 & 174 & 201 & 161 \\
\hline MAR-ABR & 102 & 131 & 189 & 169 & 226 & 164 \\
\hline MAY-JUN & 74 & 142 & 135 & 200 & 249 & 160 \\
\hline JUL-AGO & 76 & 96 & 101 & 159 & 138 & 114 \\
\hline SEP-OCT & 168 & 368 & 232 & 199 & 116 & 216 \\
\hline NOV-DIC & 195 & 184 & 239 & 246 & 128 & 198 \\
\hline \multirow[t]{2}{*}{ TOTAL } & 743 & 1.084 & 1.034 & 1.148 & 1.058 & 1.013 \\
\hline & \multicolumn{6}{|c|}{ C. E. I. P. REGINA ASSUMPTA } \\
\hline ENE-FEB-MAR & 113 & 120 & 117 & 140 & 106 & 119 \\
\hline ABR-MAY-JUN & 103 & 125 & 129 & 116 & 118 & 118 \\
\hline JUL-AGO-SEP & 52 & 55 & 84 & 71 & 83 & 69 \\
\hline OCT- NOV-DIC & 191 & 171 & 146 & 208 & 239 & 191 \\
\hline \multirow[t]{2}{*}{ TOTAL } & 460 & 470 & 477 & 535 & 545 & 497 \\
\hline & \multicolumn{6}{|c|}{ C. E. P.S. CALASANZ } \\
\hline ENE-FEB & 700 & 499 & 562 & 454 & 579 & 559 \\
\hline MAR-ABR & 321 & 672 & 547 & 511 & 502 & 511 \\
\hline MAY-JUN & 338 & 695 & 502 & 530 & 420 & 497 \\
\hline JUL-AGO & 231 & 231 & 242 & 242 & 239 & 237 \\
\hline SEP-OCT & 500 & 390 & 322 & 305 & 112 & 326 \\
\hline NOV-DIC & 312 & 534 & 402 & 500 & 388 & 427 \\
\hline TOTAL & 2.402 & 3.021 & 2.577 & 2.542 & 2.240 & 2.556 \\
\hline
\end{tabular}


Tabla 2. Indicadores de desempeño de consumo de agua de los distintos centros.

\begin{tabular}{|c|c|c|c|}
\hline INDICADORES & $\begin{array}{c}\text { C. E. I. LA } \\
\text { INMACULADA }\end{array}$ & $\begin{array}{c}\text { C. E. I. P. REGINA } \\
\text { ASSUMPTA }\end{array}$ & $\begin{array}{c}\text { C. E. P. S. } \\
\text { CALASANZ }\end{array}$ \\
\hline $\begin{array}{c}\text { CONSUMO DIARIO AGUA } \\
\text { POR USUARIO (l/usuario) }\end{array}$ & 14,8 & 7,9 & 6,2 \\
\hline $\begin{array}{c}\text { EMISIONES ANUALES CO } \\
\text { POR USUARIO (kg/usuario) }\end{array}$ & 5,4 & 2,9 & 2,3 \\
\hline $\begin{array}{c}\text { FACTURA ANUAL AGUA } \\
\text { POR USUARIO (€/usuario) }\end{array}$ & 8,5 & 5,3 & 4,9 \\
\hline
\end{tabular}

Tabla 3. Dotación de equipos consumidores de agua de los distintos centros.

\begin{tabular}{|l|c|c|c|}
\hline & $\begin{array}{c}\text { C. E. I. LA } \\
\text { INMACULADA }\end{array}$ & $\begin{array}{c}\text { C. E. I. P. REGINA } \\
\text { ASSUMPTA }\end{array}$ & $\begin{array}{c}\text { C. E. P. S. } \\
\text { CALASANZ }\end{array}$ \\
\hline Grifo sencillo & 15 & 10 & 9 \\
\hline Grifo temporizado & 0 & 4 & 43 \\
\hline Grifo monomando & 0 & 1 & 2 \\
\hline Inodoro tanque alto & 12 & 20 & 66 \\
\hline Inodoro pulsador sencillo & 3 & 3 & 0 \\
\hline Inodoro doble pulsador & 0 & 1 & 5 \\
\hline Fregadero (sin perlizador) & 2 & 5 & 13 \\
\hline Vertedero & 1 & 2 & 2 \\
\hline Urinario & 0 & 0 & 2 \\
\hline Lavavajillas & 1 & 2 & 1 \\
\hline Ducha & 3 & 0 & 20 \\
\hline Pileta laboratorio & 0 & 0 & \\
\hline
\end{tabular}

- Se estima el número de usos diario de los lavabos por cada usuario del edificio y el tiempo medio de cada uso, teniendo en cuenta el número de horas que permanecen en el mismo.

- Se estima el ratio de litros de agua por cada $\mathrm{m}^{2}$ de edificio, para su limpieza.

- Se estima el ratio de litros de agua por cada comida que se realiza en el comedor del centro docente.

- Se multiplican los consumos diarios por el número de días en los que permanece abierto el centro, para hallar el consumo anual de cada sistema.

- Se comprueba que el resultado total se ajusta al consumo medio obtenido de los datos de facturación. Se realiza el ajuste correspondiente de las estimaciones anteriores, considerando la pequeña diferencia que pueda existir como un consumo realizado por otras causas, como pueden ser las piletas de los laboratorios, las pequeñas fugas en la instalación, etc.

- A cada consumo se asocia su correspondiente gasto económico.

Los resultados del reparto realizado, se muestran en la tabla 4. Para establecer el coste asociado a cada consumo en $€$, se han aplicando los precios del agua que figuran en las facturas recogidas en cada centro docente.

En la figura 3 se refleja el reparto de consumo de agua entre los distintos equipos consumidores existentes, expresado en porcentaje con respecto al consumo total que se produce en cada centro docente.

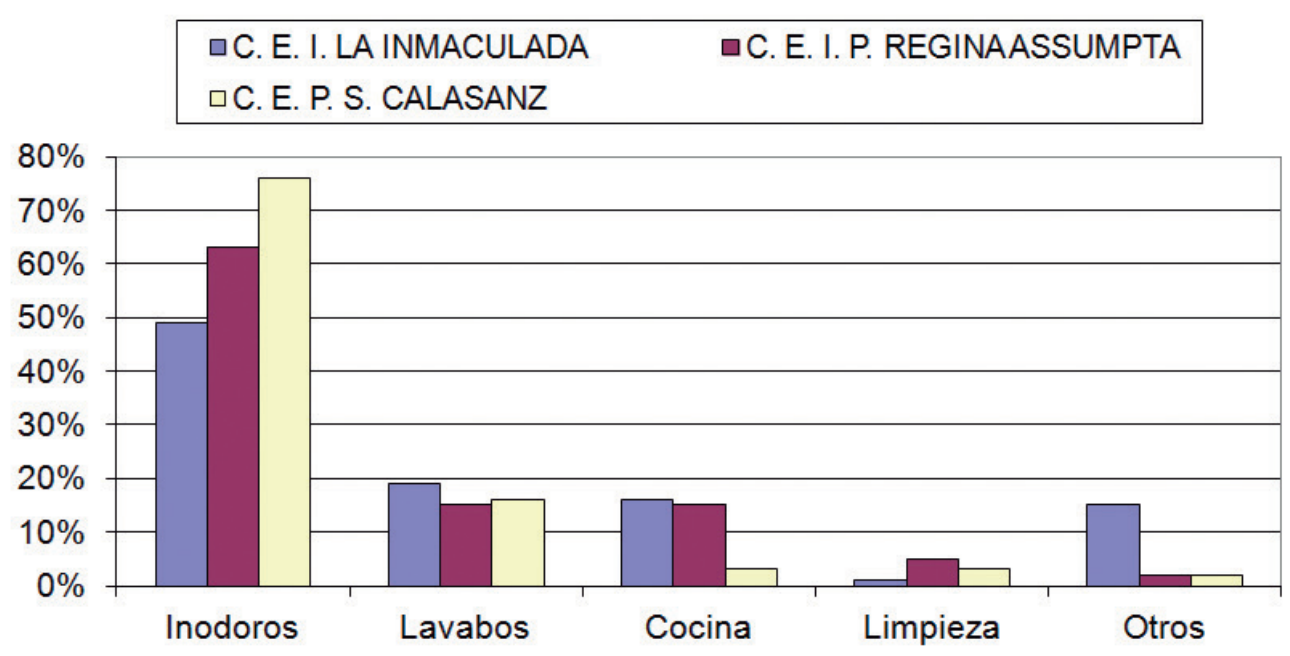

Figura 3. Reparto de consumo de agua entre los equipos expresado en porcentaje con respecto al consumo total de cada centro docente. 
Tabla 4. Reparto del consumo de agua y su coste asociado entre los distintos equipos de los centros.

\begin{tabular}{|l|c|c|c|c|c|c|}
\cline { 2 - 8 } \multicolumn{1}{c|}{} & \multicolumn{2}{c|}{ C. E. I. LA INMACULADA } & \multicolumn{2}{c|}{ C. E. I. P. REGINA ASSUMPTA } & \multicolumn{2}{c|}{ C. E. P. S. CALASANZ } \\
\cline { 2 - 8 } & $\begin{array}{c}\text { CONSUMO } \\
\text { ANUAL }\left(m^{3}\right)\end{array}$ & $\begin{array}{c}\text { FACTURA } \\
\text { ANUAL }(€)\end{array}$ & $\begin{array}{c}\text { CONSUMO } \\
\text { ANUAL }\left(m^{3}\right)\end{array}$ & $\begin{array}{c}\text { FACTURA } \\
\text { ANUAL }(€)\end{array}$ & $\begin{array}{c}\text { CONSUMO } \\
\text { ANUAL (m }\end{array}$ & $\begin{array}{c}\text { FACTURA } \\
\text { ANUAL }(€)\end{array}$ \\
\hline Inodoros & 499 & 783 & 311 & 570 & 1.937 & 4.165 \\
\hline Lavabos & 191 & 300 & 75 & 137 & 406 & 872 \\
\hline Cocina & 166 & 261 & 77 & 141 & 80 & 171 \\
\hline Limpieza & 10 & 16 & 23 & 43 & 61 & 155 \\
\hline Otros & 147 & 23 & 11 & 20 & 2.556 & 5.495 \\
\hline TOTAL & 1.013 & 1.590 & 497 & 911 & & 132 \\
\hline
\end{tabular}

A continuación, en la tabla 5, se recogen los indicadores de las distintas propuestas de mejora (P.M.)

\subsection{Discusión}

Al comparar el indicador de consumo medio de agua por usuario de cada centro docente con el valor de referencia de 4,7 litros al día por usuario $(24,25)$, se observa que todos los centros presentan un valor superior al valor de referencia, tal y como queda reflejado en la figura 4.
Con estos resultados cabe plantearse que el uso del agua que se está realizando en el centro docente es susceptible de optimizarse. No obstante, se observa que, a medida que aumenta la edad de los usuarios del centro docente, su consumo de agua disminuye. Sobre todo, los alumnos que cursan educación infantil, en sus primeros años, necesitan mayor cantidad de agua para su higiene personal.

Dado que los valores de consumo de agua obtenidos superan al valor de referencia en un $32 \%, 68 \%$ y $215 \%$ respectivamen-

Tabla 5. Indicadores de las distintas propuestas de mejora de los distintos centros docentes.

\begin{tabular}{|c|c|c|c|}
\hline & $\begin{array}{l}\text { C. E. I. LA } \\
\text { INMACULADA }\end{array}$ & $\begin{array}{l}\text { C. E. I. P. REGINA } \\
\text { ASSUMPTA }\end{array}$ & $\begin{array}{l}\text { C. E. P. S. } \\
\text { CALASANZ }\end{array}$ \\
\hline INDICADORES & \multicolumn{3}{|c|}{$\begin{array}{l}\text { P.M. 1: ECOAUDITORÍA DEL AGUA } \\
\end{array}$} \\
\hline REDUCCIÓN ANUAL DE CONSUMO (m³) & 350 & 99 & 511 \\
\hline AHORRO ECONÓMICO ANUAL (€) & 542,50 & 945,06 & $1.098,65$ \\
\hline AHORRO ANUAL DE EMISIONES $\mathrm{CO}_{2}(\mathrm{t})$ & 0,3 & 0,1 & 0,5 \\
\hline INVERSIÓN PARA IMPLANTACIÓN (€) & $\mathrm{O}$ & $\mathrm{O}$ & $\mathrm{O}$ \\
\hline $\begin{array}{c}\text { PERÍODO DE RETORNO SIMPLE } \\
\text { DE LA INVERSIÓN (años) }\end{array}$ & - & 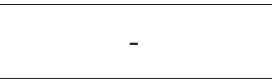 & - \\
\hline INDICADORES & \multicolumn{3}{|c|}{ P.M. 2: CONTRAPESO EN INODOROS TANQUE ALTO } \\
\hline REDUCCIÓN ANUAL DE CONSUMO (m³) & 67 & 95 & 816 \\
\hline AHORRO ECONÓMICO ANUAL (€) & 103,16 & 180,50 & $1.754,40$ \\
\hline AHORRO ANUAL DE EMISIONES $\mathrm{CO}_{2}(\mathrm{t})$ & 0,1 & 0,1 & 0,8 \\
\hline INVERSIÓN PARA IMPLANTACIÓN (€) & 174,24 & 304,92 & 958,32 \\
\hline $\begin{array}{c}\text { PERÍODO DE RETORNO SIMPLE } \\
\text { DE LA INVERSIÓN (años) }\end{array}$ & 1,7 & 1,7 & 0,5 \\
\hline INDICADORES & \multicolumn{3}{|c|}{ P.M. 3: DOBLE PULSADOR INODOROS TANQUE BAJO } \\
\hline REDUCCIÓN ANUAL DE CONSUMO (m³) & 25 & 9 & - \\
\hline AHORRO ECONÓMICO ANUAL ( $(€)$ & 38,68 & 17,10 & - \\
\hline AHORRO ANUAL DE EMISIONES $\mathrm{CO}_{2}(\mathrm{t})$ & 0,0 & 0,0 & - \\
\hline INVERSIÓN PARA IMPLANTACIÓN (€) & 180,60 & 180,6 & - \\
\hline $\begin{array}{c}\text { PERÍODO DE RETORNO SIMPLE } \\
\text { DE LA INVERSIÓN (años) }\end{array}$ & 4,7 & 10,6 & - \\
\hline INDICADORES & \multicolumn{3}{|c|}{ P.M. 4: GRIFOS TEMPORIZADOS } \\
\hline REDUCCIÓN ANUAL DE CONSUMO $\left(\mathrm{m}^{3}\right)$ & 179 & 49 & 79 \\
\hline AHORRO ECONÓMICO ANUAL (€) & 277,45 & 93,10 & 169,85 \\
\hline AHORRO ANUAL DE EMISIONES $\mathrm{CO}_{2}(\mathrm{t})$ & 0,2 & 0,0 & 0,1 \\
\hline INVERSIÓN PARA IMPLANTACIÓN (€) & 934,50 & 808,72 & 685,3 \\
\hline $\begin{array}{l}\text { PERÍODO DE RETORNO SIMPLE } \\
\text { DE LA INVERSIÓN (años) }\end{array}$ & 3,4 & 8,7 & 4,0 \\
\hline INDICADORES & \multicolumn{3}{|c|}{ P.M. 5: PERLIZADOR EN FREGADEROS } \\
\hline REDUCCIÓN ANUAL DE CONSUMO $\left(\mathrm{m}^{3}\right)$ & 106 & 59 & 34 \\
\hline AHORRO ECONÓMICO ANUAL (€) & 388,27 & 112,10 & 73,1 \\
\hline AHORRO ANUAL DE EMISIONES $\mathrm{CO}_{2}(\mathrm{t})$ & 0,3 & 0,1 & 0,0 \\
\hline INVERSIÓN PARA IMPLANTACIÓN (€) & 31,46 & 78,65 & 78,65 \\
\hline $\begin{array}{c}\text { PERÍODO DE RETORNO SIMPLE } \\
\text { DE LA INVERSIÓN (años) }\end{array}$ & 0,1 & 0,7 & 1,1 \\
\hline
\end{tabular}


口CONSUMO DIARIO AGUA POR USUARIO (I/usuario)

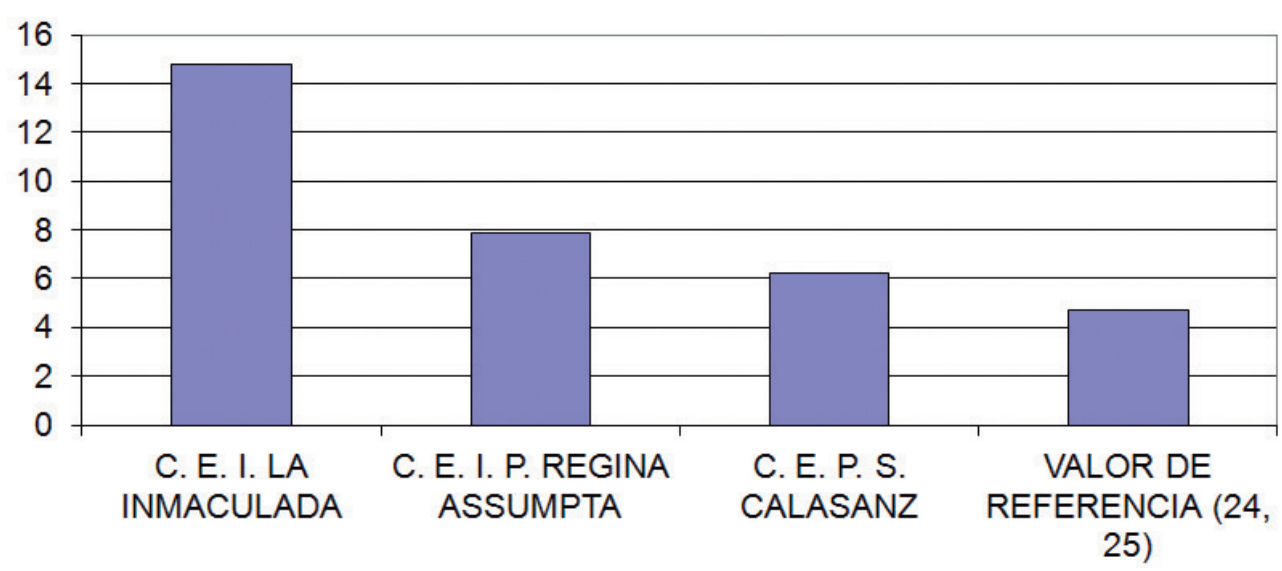

Figura 4. Reparto de consumo de agua entre los equipos, expresado en porcentaje con respecto al consumo total de cada centro docente.

te, se considera prioritario adoptar la propuesta de mejora 1 , que incide sobre la utilización del recurso agua por parte de los usuarios de los centros docentes.

En cuanto al reparto del consumo de agua entre los distintos equipos, cabe destacar que el principal consumo se realiza en los inodoros. En dichos sanitarios se consume un porcentaje entre el $65 \%$ y el $75 \%$ del total de agua consumida, a excepción del C.E.I. La Inmaculada, donde dicho porcentaje es del $50 \%$. Esto se debe a que en dicha escuela el consumo de agua en el apartado "otros" crece al 15\%, mientras que en el resto de centros permanece en el $2 \%$, por el agua usada en las duchas para la higiene de niños que están empezando a controlar sus esfínteres.

En este sentido las inversiones que se acometan para reducir el consumo de agua en los inodoros cabe esperar que tendrán una mayor repercusión en cuanto a la cantidad de agua ahorrada.

Seguidamente nos encontramos con que el consumo de agua en lavabos y en la cocina presenta valores similares en torno al 15\% del total de agua consumida, a excepción del C.E.P.S. Calasanz donde los valores en la cocina bajan al $2 \%$, debido a que tiene un horario continuo, lo que implica que el número de alumnos que utilizan el comedor es mucho más reducido que en el resto de casos.

Por este motivo, las medidas que se proponen en los lavabos y en la cocina cabe esperar que tengan una menor incidencia en cuanto al ahorro de agua que producen, frente a las medidas estudiadas anteriormente.

Debido a que el porcentaje de agua que se utiliza en la limpieza de los centros y en otros es tan poco relevante, con valores comprendidos entre el $2 \%$ y el $5 \%$, las reducciones que se podrían obtener en estos apartados resultan poco significativas, por lo que se descarta su estudio.

En la figura 5 se pueden observar los ahorros de agua en $\mathrm{m}^{3}$ que se obtienen con la aplicación de las diferentes propuestas de mejora en los distintos centros. El ahorro de emisiones de $\mathrm{CO}_{2}$ y el ahorro económico que suponen dichas medidas mantienen una relación lineal con el ahorro de agua.

\section{口C. E. I. LA INMACULADA \\ 口C. E. I. P. REGINAASSUMPTA \\ $\square$ C. E. P. S. CALASANZ}

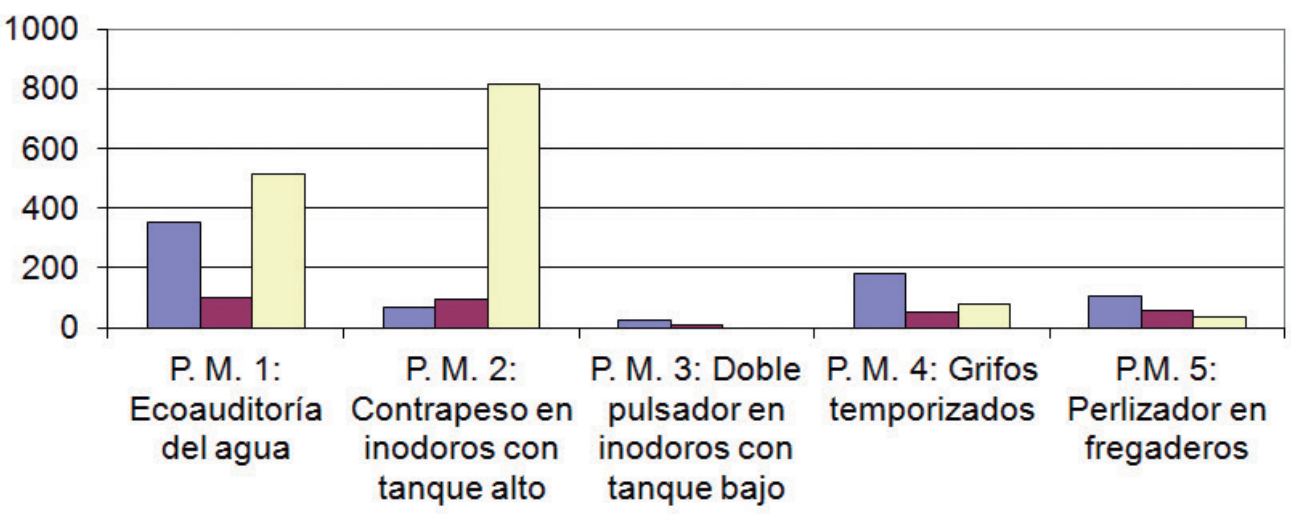

Figura 5. Ahorro de agua en $\mathrm{m}^{3}$ que se obtiene con la aplicación de las propuestas de mejora (P. M.) en los distintos centros. 
Los resultados que se obtienen difieren de los resultados esperados en función del porcentaje de consumo que se produce en cada sistema consumidor de agua.

En el C.E.I. La Inmaculada, el ahorro que se obtiene en los lavabos duplica al ahorro que se produce en los inodoros. Esto es debido a dos causas. La primera es que el edificio se construyó en el año 2002 y los inodoros que tiene instalados no despilfarran tanta agua como los existentes en edificios más antiguos. La segunda causa es que se ha detectado que el 15\% de los grifos instalados suministran un caudal seis veces el valor del resto de grifos.

El C.E.I.P. Regina Assumpta ofrece unos resultados acordes a lo que cabría esperar en función del porcentaje de consumo que se produce en cada sistema consumidor de agua.

En el C.E.P.S. Calasanz el ahorro que se obtiene en los inodoros es mayor que lo que cabría esperar en función de su porcentaje de consumo. Esto es debido a que la media de las cisternas instaladas sobrepasa en un 63\% la capacidad de almacenaje de agua de las cisternas actuales.

Para comparar los resultados de las inversiones necesarias para implantar las propuestas de mejora, se utiliza el indicador de intensidad económica entendido como el resultado de dividir la inversión a realizar en $€$ entre la facturación anual en $€$ y se expresa en \%, tal y como se refleja en la figura 6 .

Se puede observar que la propuesta de mejora 1, ecoauditoría del agua, no tiene un coste económico dado que se lleva a cabo con los medios humanos del centro, al realizarse como una actividad educativa.

Las propuestas de mejora 2 y 3, relativas a los inodoros, suponen un desembolso alrededor del $20 \%$ de la facturación anual, salvo en el C.E.I.P. Regina Assumpta que alcanza valores cercanos al 50\%. Esto es debido a que dicho centro tiene una mayor superficie por número de alumnos y, consecuentemente, mayor número de inodoros por alumno que el resto de centros.

La propuesta de mejora 4, relativa a los lavabos, presenta mayor dispersión con valores cercanos al 60\% en el C.E.I. La Inmaculada, 90\% en el C.E.I.P. Regina Assumpta y 10\% en el C.E.P.S. Calasanz. El mayor valor obtenido en el C.E.I.P. Regina Assumpta tiene la misma explicación que con los inodoros: un mayor número de lavabos por alumno del centro.

La propuesta de mejora 5, perlizador en fregaderos, supone una inversión por debajo del 10\% de la facturación en todos los centros.

En general el C.E.P.S. Calasanz presenta los menores valores de intensidad económica en cuanto a las inversiones a realizar para implantar las medidas, debido al factor de escala: tiene 5 veces más alumnos que el resto de centros.

Los resultados de los períodos de retorno simple de la inversión, expresados en años, de las distintas propuestas de mejora aplicadas a los distintos centros docentes se pueden observar en la figura 7.

En primer lugar cabe destacar que todas las propuestas de mejora presentan un período de retorno simple de la inversión inferior a 5 años, lo que hace que su puesta en marcha resulte muy atractiva, a excepción de la incorporación de doble pulsador en los inodoros con tanque bajo y de grifos temporizados en el C.E.I.P. Regina Assumpta. Esto es debido, como en el análisis de resultado efectuado anteriormente, a que dicho centro tiene una mayor superficie por número de alumnos y, consecuentemente, mayor número de inodoros y lavabos por alumno que el resto de centros.

Adicionalmente, las propuestas de mejora 2: Contrapeso en inodoros con tanque alto y 5: Perlizador en fregaderos, presentan un período de retorno simple de la inversión inferior a 2 años, lo que hace que su puesta en marcha resulte extremadamente atractiva.

Por último, la propuesta de mejora 1: Ecoauditoría del agua, no presenta período de retorno simple de la inversión, dado que, al tratarse de un centro educativo, su implantación no supone un coste adicional. Lo que no quiere decir que no suponga un coste de tiempo, de recursos humanos y de material, sino que dichos costes se pueden repercutir a la actividad educativa que se desarrolla en el centro docente. No supone un coste adicional porque si dichos recursos no se emplean en realizar la propuesta sugerida, se van a emplear en realizar otra actividad educativa. Su implantación depende úni-

\section{口C. E. I. LA INMACULADA \\ 口C. E. I. P. REGINAASSUMPTA \\ $\square$ C. E. P. S. CALASANZ}

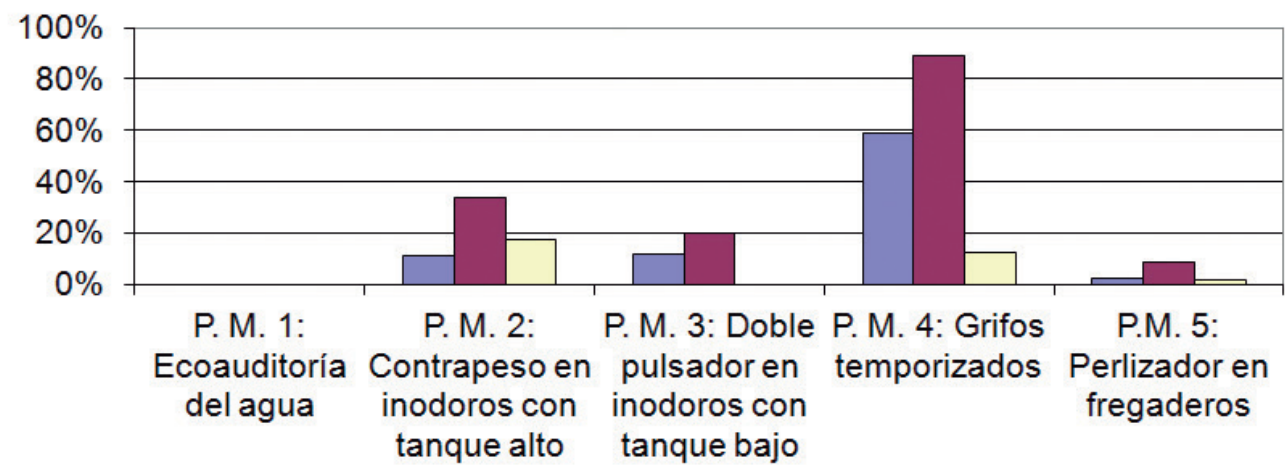

Figura 6. Intensidad económica de la inversión expresada en \% sobre la facturación anual de las propuestas de mejora (P. M.) en los distintos centros. 


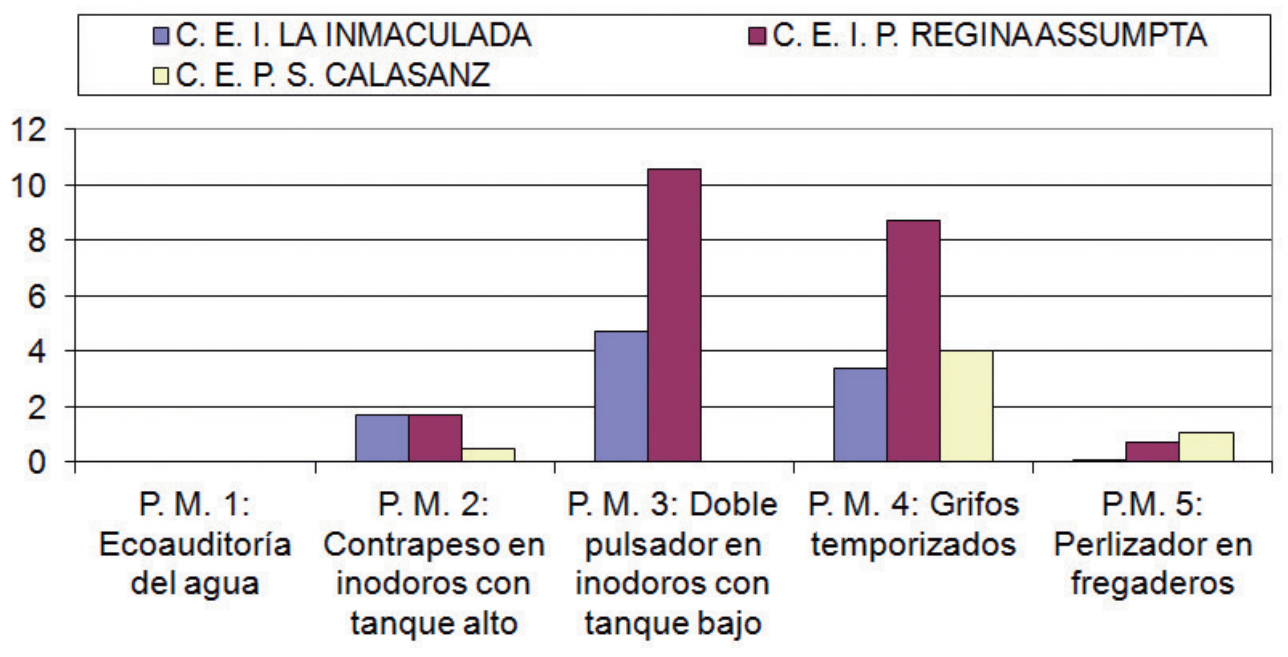

Figura 7. Período de retorno simple de la inversión, expresado en años, de las distintas propuestas de mejora (P. M.) en los distintos centros.

camente de que así se decida, sin que resulte necesaria la búsqueda de financiación adicional. Es importante la implicación del usuario en cualquier intento de mejorar la eficiencia en el uso de la energía y de los recursos como el agua. Las propuestas basadas únicamente en la tecnología que dejan de lado la participación de los usuarios no tienen en cuenta una parte importante del proceso. Los edificios no usan energía (ni agua), las personas lo hacen (33).

De esta manera, si el criterio para la puesta en marcha de las propuestas de mejora fuera empezar por aquellas de mayor rentabilidad económica, sería aconsejable poner en marcha la P.M.1, en primer lugar, para continuar por las P.M. 5 y 2 a continuación y terminar con las P.M. 4 y 3.

\section{CONCLUSIONES}

Dejar de lado el estudio del consumo de agua mientras se analiza el desempeño energético de los centros docentes supone una limitación de enfoque, dado que, como se ha podido comprobar, el consumo de agua lleva irremediablemente asociado un consumo de energía. Por ello, introducir el concepto de metabolismo en los edificios, a la hora de su estudio, aporta un rango de conclusiones mayor.

Se observa que las medidas que se adopten encaminadas a reducir el consumo de agua son, en primer lugar necesarias, dado que el consumo que se realiza en los centros estudiados está alejado de los valores medios de edificios del mismo uso.

Además, gracias a la metodología de la auditoría energética operativa que se ha empleado, se ha logrado obtener resultados en cuanto al reparto del consumo de agua entre los distintos sistemas consumidores y se ha logrado cuantificar de cada propuesta de mejora, la reducción de consumo, el ahorro económico, el ahorro de emisiones de $\mathrm{CO}_{2}$, la inversión para su implantación, así como el período de retorno simple de la inversión.

La reducción de emisiones de $\mathrm{CO}_{2}$ arroja cifras poco relevantes, entre 0,9 y 1,4 toneladas de $\mathrm{CO}_{2}$, si se implantasen la totalidad de las medidas. Aunque todo suma, la reducción de emisiones resulta menos relevante que la que se produce con medidas relacionadas con el consumo de energía para calentar los colegios o el consumo eléctrico dentro de los mismos.

Los resultados obtenidos permiten al gestor del centro docente tener un criterio informado que ayude en el proceso de toma de decisión acerca de las medidas a implantar, lo necesarias que resultan, las inversiones que precisan y el orden en el que acometerlas.

El mayor ahorro de agua se obtiene con el trabajo que se propone realizar con los usuarios de los centros educativos a través de la ecoauditoría del agua, con ahorros comprendidos entre el $20 \%$ y el $35 \%$.

En cuanto a los equipos en los que se consume agua en los centros docentes, se consigue mayor ahorro de agua interviniendo en los inodoros, con ahorros comprendidos entre el $10 \%$ y el $30 \%$. Los siguientes aparatos en los que se consigue mayor ahorro de agua son los lavabos, con ahorros comprendidos entre el $5 \%$ y el $20 \%$.

En cuanto al tipo de colegios, se ha observado que donde más agua se consume es en el centro de educación infantil, debido a la mayor necesidad de aseo que presentan los alumnos al ser más pequeños. En estos centros conviene incidir en los hábitos de consumo con los usuarios. En los colegios de educación primaria y en los que cuentan con educación secundaria tiene mayor peso el consumo de agua en los inodoros. Aunque en los colegios con educación primaria, debido a que hay más alumnos que comen en el comedor del colegio, también se podría actuar en los fregaderos de las cocinas, teniendo esta propuesta de mejora el menor impacto de todas las estudiadas.

$\mathrm{Al}$ igual que se ha analizado la relación entre el uso del agua y los intercambios de energía que se producen en los edificios, se puede seguir avanzando en la investigación realizando el análisis de otros flujos de materia e información, de manera que se establezca la relación entre dichos flujos y el consumo de energía que llevan aparejados. De esta manera, se pueden estudiar medidas que reduzcan la emisión de gases efecto invernadero mediante la mejora de la eficiencia energética en el resto de procesos metabólicos que se producen en el edificio más allá de los intercambios directos de energía. 


\section{REFERENCIAS}

(1) Real Academia Española (19 de septiembre de 2018). Diccionario de la lengua española (23. ${ }^{\mathrm{a}}$ ed.). Recuperado de http://dle.rae.es/srv/fetch?id=P4EcqNv

(2) Hermanowicz, S. W. \& Asano, T. (1999). Abel Wolman's "The Metabolism of Cities" revisited: A case for water recycling and reuse. Water Science and Technology, 4O(4-5): 29-36. https://doi.org/10.2166/wst.1999.0571

(3) Kennedy, C., Cuddihy, J. \& Engel-Yan, J. (2007). The changing metabolism of cities. Journal of industrial ecology, 11(2): 43-59. https://doi.org/10.1162/jie.2007.1107

(4) Díaz Álvarez, C.J. (2014). Metabolismo urbano: herramienta para la sustentabilidad de las ciudades. Interdisciplina, 2(2): 51-70. https://doi.org/10.22201/ceiich.24485705e.2014.2.46524

(5) Dobbelsteen, A. van den (2010). Green Dream. En How Cities can outsmart nature. Rotterdam: Nai Publishers.

(6) Wadel, G., Avellaneda, J. \& Cuchí, A. (2010). La sostenibilidad en la arquitectura industrializada: cerrando el ciclo de los materiales. Informes de la Construcción, 62(517): 37-51. https://doi.org/10.3989/ic.09.067

(7) Braungart, M. \& McDonough. W. (2005). De la cuna a la cuna. Rediseñando la forma en que hacemos las cosas. Madrid: McGraw-Hill.

(8) Aranda, A. et al. (2016). El análisis del ciclo de vida como herramienta de gestión empresarial. Madrid: Fundación Confemetal.

(9) García-Erviti, F., Armengot-Paradinas, J. \& Ramírez-Pacheco, G. (2015). El análisis del coste del ciclo de vida como herramienta para la evaluación económica de la edificación sostenible. Estado de la cuestión. Informes de la Construcción, 67(537): e056. https://doi.org/10.3989/ic.12.119

(10) Moore, R. (2012). Definitions of fuel poverty: Implications for policy. Energy Policy, 49: 19-26. https://doi.org/10.1016/j. enpol.2012.01.057

(11) Boardman, B. (1991). Fuel Poverty: From Cold Homes to Affordable Warmth. London: Belhaven Press.

(12) Luxán, M., Sánchez-Guevara, C., Román, E., Barbero, M. \& Gómez, G. (2017). Re-habilitación exprés para hogares vulnerables. Soluciones de bajo coste. Barcelona: Fundación Gas Natural Fenosa.

(13) Sánchez-Guevara, C., Mavrogianni, A. \& Neila, F. J. (2017). On the minimal thermal habitability conditions in low income dwellings in Spain for a new definition of fuel poverty. Building and Environment, 114: 344-356. https://doi. org/10.1016/j.buildenv.2016.12.029

(14) Luxán, M., Gómez, G. \& Román, E. (2015). Cuentas energéticas no habituales en edificación residencial. Informes de la Construcción, 67(EXTRA-1): mo28. https://doi.org/10.3989/ic.14.059

(15) Mestre, N. (2014) Ciudad y oportunismo energético. Solapes y vacíos entre la ordenanza urbanística y los códigos técnicos en materia de energía. Madrid: CONAMA.

(16) Gómez, E. (2015). Direct and indirect water supply: an energy assessment. Procedia Engineering, 119: $1088-1097$. https://doi.org/10.1016/j.proeng.2015.08.941

(17) Kenway, S. (2013). Managing water-related energy in future cities - a research and policy roadmap. Journal of Water and Climate Change, 4(3): 161-175. https://doi.org/10.2166/wcc.2013.063

(18) Scott, C.A. (2016). The Urban Water-Energy Nexus: Building Resilience for Global Change in the Urban Century. In Hettiarachchi H., Ardakanian R. (eds) Environmental Resource Management and the Nexus Approach, (pp. 113-140). Springer, Cham. https://doi.org/10.1007/978-3-319-28593-1_5

(19) Millán, J. (2015). Agua y energía, Buenos Aires: Corporación Andina de Fomento.

(20) Gallego, J., Larrumbide, E. \& Bedoya, C. (2017). The incorporation of the study into water consumption in energy audits in schools. Revista de la construcción, 16(3): 361-373. https://doi.org/10.7764/rdlc.16.3.361

(21) Schneider, D. (2011). Hybrid nature. Sewage treatment and the contradictions of the industrial ecosystem. Cambridge: MIT Press.

(22) Ibáñez, D. \& Katsikis, N. (2011). New Geographies o6. Grounding Metabolism. Cambridge: Harvard University Graduate School of Design.

(23) Gallego, J. (2018). La auditoría energética operativa en centros docentes como herramienta para mejorar el ahorro y la eficiencia energética. (Tesis doctoral). Universidad Politécnica de Madrid.

(24) Barón, A., González, C. (2003). La ecoauditoría del agua en tu centro educativo. Guía para el profesorado. Palma de Mallorca: Fundación Ecología y Desarrollo.

(25) CEPYME ARAGÓN (3 de octubre de 2018). Guía práctica sobre ahorro de agua. Recuperado de https://www.camarazaragoza.com/medioambiente/docs/publicaciones/publicacion98.pdf

(26) Ministerio de Industria, Energía y Turismo, Ministerio de Fomento. (2016). Factores de emisión de CO ${ }_{2}$ y coeficientes de paso a energía primaria de diferentes fuentes de energía final consumidas en el sector de edificios en España.

(27) Gallego, J., Larrumbide, E. \& Bedoya, C. (2017, 8-10 marzo). La auditoría energética y el consumo de agua. En Lopez, O. et al. (Ed.) Actas del $2^{\circ}$ Congreso Internacional de Innovación Tecnológica en Edificación (pp. 60-61). Madrid: Universidad Politécnica de Madrid.

(28) Martín, L. (2014, 6-7 mayo). Reducir el consumo de energía mediante un uso sostenible del agua. Agua y energía, un binomio indisoluble. En Libro de Comunicaciones II Congreso de Edificios de Energía Casi Nula (pp. 64-66). Madrid: Grupo Tecma Red.

(29) Cabrera, E., Pardo, M. A., Cabrera, E. Jr. \& Cobacho, R. (2010). Agua y energía en España. Un reto complejo y fascinante. Ingeniería del Agua, 17 (3): 235-246. https://doi.org/10.4995/ia.2010.2976

(30) Martínez, F. J. (2011). Estudio de la huella energética del abastecimiento urbano de agua de la provincia de Almería. (Trabajo de Investigación del Máster Agua y Medio Ambiente en Áreas Semiáridas AQUARID). Universidad de Almería.

(31) Consejería de Educación, Juventud y Deporte (2017). Datos y cifras de la educación 2016-2017. 
(32) Instituto Nacional de Estadística (26 de septiembre de 2018). Censo de población y viviendas. Recuperado de https:// www.ine.es

(33) Janda, K.B. (2011). Buildings don't use energy: people do. Architectural science review, 54(1): 15-22. https://doi. org/10.3763/asre.2009.0050 